\title{
Modeling Prominence Formation in 2.5D
}

\author{
X. Fang, C. Xia and R. Keppens
}

Centre for mathematical Plasma Astrophysics, Department of Mathematics, KU Leuven

\begin{abstract}
We use a 2.5-dimensional, fully thermodynamically and magnetohydrodynamically compatible model to imitate the formation process of normal polarity prominences on top of initially linear force-free arcades above photospheric neutral lines. In magnetic arcades hosting chromospheric, transition region, and coronal plasma, we perform a series of numerical simulations to do a parameter survey for multi-dimensional evaporation-condensation prominence models. The investigated parameters include the fixed angle of the magnetic arcade, the strength and spatial range of the localized chromospheric heating.
\end{abstract}

Keywords. magnetohydrodynamics $(\mathrm{MHD})$ - Sun: corona — Sun: filaments, prominences

Prominences, a common feature in active and quiet solar regions, represent huge structures of cold $\left(\approx 10^{4} \mathrm{~K}\right)$ and dense $\left(10^{10}-10^{11} \mathrm{~cm}^{-3}\right)$ plasma in the solar atmosphere (Tandberg-Hanssen (1995)). They are hosted by strong and complex dip-shaped magnetic field configurations, usually above the magnetic polarity inversion lines. However, the magnetic field topology of prominences is still poorly understood, although observations indicate it is mainly horizontal, with an acute angle with respect to the main axis of prominences (Bommier \& Leroy (1998)). Prominences attracted plenty of theoretical studies to address different aspects of them, such as formation and eruption. Especially considering the formation of prominences, recently Xia et al. (2012) realized a 2.5D simulation of in situ formation of a filament in a sheared magnetic arcade, with chromospheric evaporation plus coronal condensation, using the MPI-parallelized Adaptive Mesh Refinement (AMR) Versatile Advection Code (Keppens et al. (2012)).

Here we present a parameter study based on Xia et al. (2012). We follow the setup in Xia et al. (2012) as a 2.5D thermodynamic MHD model on a 2D domain of size 40 by $50 \mathrm{Mm}$ (in $x-y$ ), but now adopt a linear force-free magnetic field characterized by a constant angle $\theta_{0}$ (as in Fang et al. (2013)) as the initial magnetic field topology. The background heating rate decays exponentially with height, which helps to obtain a self-consistent thermally structured corona at first, and a relatively strong additional heating near the chromosphere injects energy and evaporates the plasma. We choose different angles $\left(\theta_{0}=30^{\circ}, 45^{\circ}\right)$ for the initial linear force-free magnetic field topology. In the simulations, we regulate the energy input from additional chromospheric heating to reach the same value among the models with different angles. We also study models with different magnetic field strength, different energy input heating scale and different spatial ranges of the additional chromospheric heating.

\section{Results and discussion}

In the left panel of Fig.1, simulations of two representative models with different angles for the arcade magnetic field are compared, showing the evolution of the prominence mass. They indicate that after the appearance of cool plasma, an approximate linear relationship with time is found and the growth rates of the condensations in these models are similar. We infer this is because of the regulated same energy input from the additional chromospheric heating. By analyzing the growth rates of accumulated prominence mass 

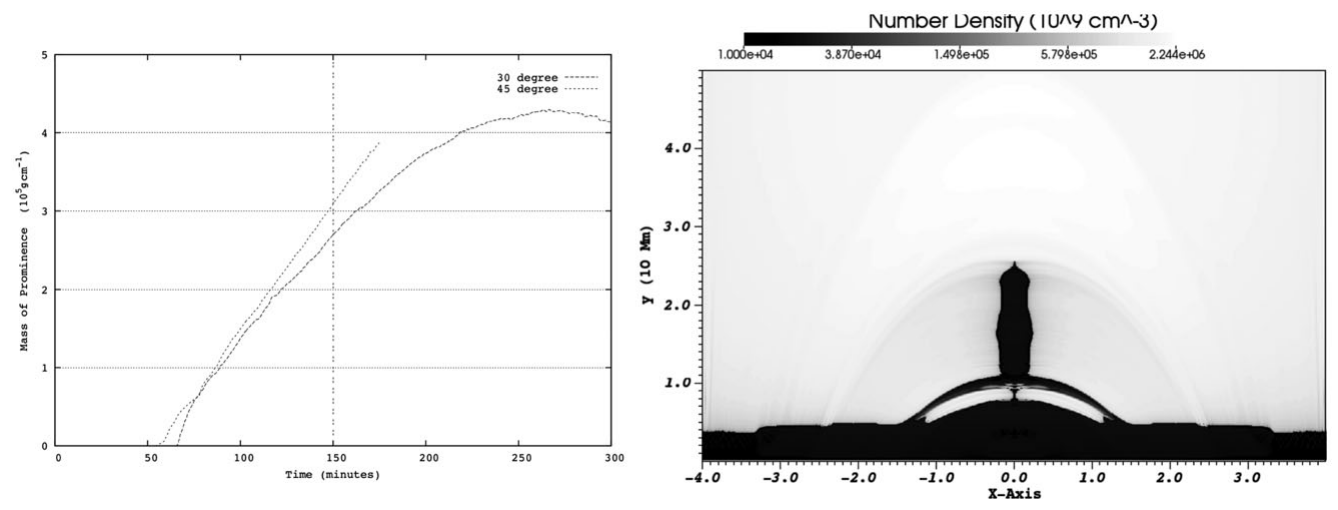

Figure 1. Left Panel: Temporal evolution of the prominence mass of models with two different angles of magnetic field. The vertical dashed line at $\mathrm{t}=150 \mathrm{~min}$ shows the moment when prominences begin to drag extra mass.; Right Panel: The density snapshot at $t=240 \mathrm{~min}$.

in models with different parameters (a total of 10 models were intercompared, not shown in Fig. 1), we infer that these growth rates are basically determined by the energy input from additional chromospheric heating, although the time of formation and the heights of the first condensations can differ. Furthermore, by adopting different heating lengthscales and spatial ranges of the additional chromosphere heating with the same angle $\left(\theta_{0}=30^{\circ}\right)$ of magnetic field topology, simulations still demonstrate that the growth of condensations display nearly linear relationship with time and positively correlate with total energy inputs from the additional heating. In models hosting different magnetic field strength, we did not find any obvious relationship between the growth rates of the condensations and the magnetic field strength.

Some of our modeled prominences develop additional internal structure, with the side boundaries of the prominence resembling sawteeth, when the magnetic field of the arcade is strong. Indeed, when the lateral growing prominence can not bend the arched loops fast enough, segments of the prominence body residing in self-created magnetic dips fall down to the chromosphere along the arched loops. This drags extra mass from inside the magnetic dips to stream down until all prominence mass in the affected loops drains to the chromosphere. Consecutively, the evacuated loops reform condensations, and this phenomenon propagates from lower to higher loops. This realizes a down-streaming channel adjacent to an up-streaming channel, reforming the prominence as it rises, and we suggest these long-lived streams connecting the prominence and the chromosphere resemble the barbs of prominences (Fig. 1, right panel). They also shed light on the mass recycling puzzle of prominences in general.

\section{References}

Bommier, V. \& Leroy, J. L. 1998, IAU Colloq. 167: NPSP, 150, 434

Fang, X., Xia, C., \& Keppens, R. 2013, Ap. Lett., 771, L29

Keppens, R., et al. 2012, JCP, 231, 718

Xia, C., Chen, P. F., \& Keppens, R. 2012, Ap. Lett., 748, L26

Tandberg-Hanssen, E. 1995, Science, 269, 111 